\title{
Acetylation of Yeast Histone H4 Lysine 16: A Switch for Protein Interactions in Heterochromatin and Euchromatin
}

\author{
C.B. Millar, S.K. Kurdistani, and M. Grunstein \\ Department of Biological Chemistry, UCLA School of Medicine and the Molecular Biology Institute, \\ Los Angeles, California 90095
}

\begin{abstract}
Histone acetylation is an evolutionarily conserved phenomenon that can alter chromatin structure and activity. The basic unit of chromatin, the nucleosome, contains two molecules of each of the core histones, H3, H4, H2A, and $\mathrm{H} 2 \mathrm{~B}$, wrapped by DNA. Each core histone has a central hydrophobic domain that contributes to the histone-histone and histone-DNA contacts that form the basis of the structural organization of the nucleosome (Luger et al. 1997). Extending from the nucleosomal core, the charged amino- and carboxy-terminal tail domains of the histones are free to interact with linker DNA, adjacent nucleosomes, and other chromosomal proteins (Zheng and Hayes 2003). These tail domains can be modified by acetyltransferases, methyltransferases, and kinases, thus altering their chemical characteristics and, consequently, those of the chromatin fiber.
\end{abstract}

Acetylation of lysine residues by histone acetyltransferases (HATs) is the most prevalent type of histone modification (Zhang et al. 2003) and can be rapidly reversed through the action of histone deacetylases (HDACs). Addition of an acetyl group neutralizes the positive charge of a lysine, and this change in charge can affect the in vitro interactions of histone tails with DNA, adjacent nucleosomes, and other chromatin components, ultimately altering the higher-order folding of chromatin (Tse et al. 1998; Zheng and Hayes 2003). However, the extent to which such charge-induced structural changes occur in vivo is unclear. A complementary view of the function of acetylation is based on its role in regulating the binding of proteins to the histone tails. It has been shown in genetic and biochemical studies that interactions of certain chromosomal regulatory proteins with histone tails are dependent on the histones' acetylatable lysines (Johnson et al. 1990; Hecht et al. 1995; Jacobson et al. 2000; Corona et al. 2002; Kasten et al. 2004). For example, Sir3, a chromosomal regulator that binds to histones and organizes heterochromatin structure in yeast, requires the deacetylation of H4-K16 for its interaction with chromatin (Johnson et al. 1990; Hecht et al. 1995) and preferentially binds to a completely deacetylated $\mathrm{H} 4$ amino terminus in vitro (Carmen et al. 2002). In contrast, proteins containing bromodomains interact preferentially with acetylated histone tails via a conserved hydrophobic pocket within the bromodomain that selectively binds acetyl-lysine residues (Dhalluin et al. 1999). SANT domains also interact with histones and may prefer to bind unacetylated histone tails, although the structural basis for this discrimination is unknown (Boyer et al. 2002; Yu et al. 2003). Numerous chromatin remodeling complexes contain at least one bromo- and/or SANT domain, indicating that the regulation of binding by acetylation and deacetylation may be a common mechanism for controlling protein-protein interactions within chromatin. In this paper we will discuss the role of H4-K16 acetylation in yeast heterochromatin and euchromatin as a switch to affect the binding of regulatory proteins to chromatin.

\section{HISTONE H4 ACETYLATION SITES ARE NONREDUNDANT}

The lysine residues at positions $5,8,12$, and 16 (K5, $\mathrm{K} 8$, K12, and K16) within the amino-terminal tail of $\mathrm{H} 4$ are acetylated in Saccharomyces cerevisiae and all other species examined to date. In yeast, this region of H4 is important for a number of cellular processes including chromatin assembly (Ma et al. 1998), heterochromatic silencing (Kayne et al. 1988), activation of inducible genes (Durrin et al. 1991), repression of basal transcription (Lenfant et al. 1996), and double-strand break repair (Bird et al. 2002). In all of these processes, there is a distinction between the roles of sites K5, K8, and K12 (and their acetylation and/or deacetylation) and that of K16. For example, sites $\mathrm{K} 5, \mathrm{~K} 8$, and $\mathrm{K} 12$ are redundant with each other, but not with K16, for chromatin assembly (Ma et al. 1998). Correspondingly, mutations in K16 have a uniquely strong effect on repression of heterochromatin at the silent mating loci (Johnson et al. 1990; Megee et al. 1990; Park and Szostak 1990) and telomeres (Aparicio et al. 1991). The roles for acetylated sites in DNA doublestrand break repair are not well-characterized, but mutants in $\mathrm{K} 5, \mathrm{~K} 8$, and $\mathrm{K} 12$ behave similarly, while $\mathrm{K} 16$ is less important in this process (Bird et al. 2002). Taken together, these results suggest that K16 has functions that are distinct from the other acetylated sites on $\mathrm{H} 4$.

\section{ROLE OF H4-K16 ACETYLATION AND DEACETYLATION IN THE FORMATION AND SPREADING OF TELOMERIC HETEROCHROMATIN}

The H4 amino-terminal tail plays an important role in heterochromatic silencing at yeast telomeres and matingtype loci (Kayne et al. 1988; Aparicio et al. 1991). Mutations of individual lysine residues in the $\mathrm{H} 4$ tail show that, 


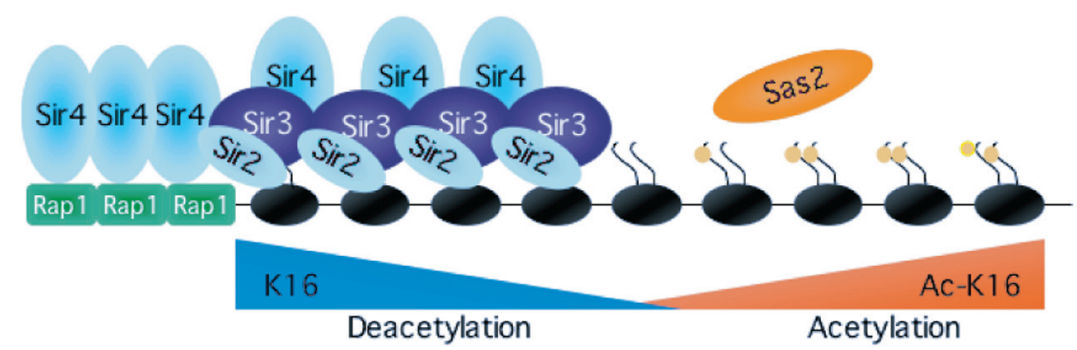

Figure 1. Initiation and spreading of telomeric heterochromatin and its regulation by H4-K16 (de)acetylation. Rap1 determines where heterochromatin initiates by binding to cis-acting DNA sequences and recruiting Sir4. This step is independent of H4-K16, but all subsequent events require the unacetylated K16 residue (Hoppe et al. 2002; Luo et al. 2002). These events include recruitment of Sir2 and Sir3 to the initiation site and subsequent spreading of Sir2, Sir3, and Sir4 along the chromosome. The nucleosomes are shown as black circles, with the $\mathrm{H} 4$ amino termini extending from them. The interaction between Sir3 and the histones requires H4-K16 deacetylation, which is achieved through the action of Sir2. The acetylation of H4-K16 (orange circles) by Sas 2 prevents Sir protein binding and therefore establishes a boundary to the spreading of heterochromatin.

of the four acetylated sites, K16 is critical for silencing whereas K5, K8, and K12 are much less important (Kayne et al. 1988; Johnson et al. 1990; Megee et al. 1990; Park and Szostak 1990). The H4 amino terminus, and K16 in particular, is required for the binding of Sir3, one of the components of the SIR (silent information regulator) silencing complex that spreads from initiation sites to form heterochromatin (Johnson et al. 1990; Hecht et al. 1996). At the right arm of chromosome VI, the spreading of the SIR complex extends only as far as $\sim 3$ kilobases from the telomere, despite the absence of a discrete DNA boundary element. In fact, specific changes in chromatin components, both within the telomeric heterochromatin and in the subtelomeric euchromatin, regulate the extent of spreading of the SIR proteins from the telomeres into the subtelomeric chromatin (van Leeuwen et al. 2002; Kristjuhan et al. 2003; Ladurner et al. 2003; Meneghini et al. 2003; $\mathrm{Ng}$ et al. 2003). One such change is the deacetylation (in telomeric heterochromatin) and acetylation (in subtelomeric euchromatin) of K16 by the Sir2 HDAC and Sas2 HAT, respectively (Kimura et al. 2002; Suka et al. 2002). The role of H4-K16 in initiation and spreading of telomeric heterochromatin is illustrated in Figure 1.

$\mathrm{K} 16$ is part of a larger stretch of basic amino acids (residues 16-19) that are required for Sir3 binding in vivo and in vitro (Johnson et al. 1990; Hecht et al. 1995, 1996). The charge of K16 is crucial, since substitution with an uncharged residue or increased acetylation of K16 reduces Sir3 binding to the H4 amino terminus (Johnson et al. 1990; Carmen et al. 2002; Kimura et al. 2002). In this context, K16 acetylation and deacetylation may function as an electrostatic switch that prevents or enables binding of Sir3 to a larger domain and thus controls the formation and boundaries of heterochromatin.

\section{GLOBAL H4-K16 ACETYLATION}

Heterochromatin accounts for a relatively small proportion of the yeast genome (if the amount of heterochromatin at chromosome VI-R is indicative of other chromosomes). However, H4-K16 acetylation is a highly abundant modification, with $\sim 80 \%$ of histone $\mathrm{H} 4$ molecules having an acetyl group on K16 (Clarke et al.
1993; Smith et al. 2003). The function of K16 acetylation is therefore unlikely to be restricted to the formation of a boundary between telomeric heterochromatin and euchromatin. Indeed, in Drosophila, H4-K16 acetylation plays a key role in transcriptional activation of the male $\mathrm{X}$ chromosome for dosage compensation (Akhtar and Becker 2000). In yeast, K16 may similarly modulate transcription regulation in euchromatic regions. It is known that the acetylation sites at the amino terminus of $\mathrm{H} 4$ are required for the activation of a number of inducible promoters (Durrin et al. 1991) and for the steady-state expression of many genes (Sabet et al. 2003), but the specific role of K16 in transcription is otherwise unclear. As part of a systematic analysis of the acetylation status of lysines in the four core histones, we have obtained information about the genome-wide acetylation profile of 11 of these sites, including K16. This information has allowed us to examine the relationships between the acetylated sites and between each site and transcriptional activity (Kurdistani et al. 2004).

\section{PATTERNS OF HISTONE ACETYLATION THROUGHOUT THE GENOME}

The development of antibodies that are highly specific to each acetylated lysine has allowed us to examine the relative acetylation levels of specific sites at a number of heterochromatic and euchromatic loci by chromatin immunoprecipitation (ChIP) (Suka et al. 2001; Wu et al. 2001). Moreover, the fusion of ChIP analysis and microarrays (Ren et al. 2000; Iyer et al. 2001) has enabled genome-wide studies of the targets of histone deacetylases in yeast and, most recently, of the patterns of acetylation in wild-type cells (Kurdistani et al. 2002, 2004; Robyr et al. 2002).

Using antibodies specific for 11 different acetylated lysines on the four core histones, we isolated fragments of DNA cross-linked to histones acetylated on particular sites. These DNA fragments were then amplified and used to probe microarrays spotted with either 6200 open reading fames (ORFs) or 6700 intergenic regions (IGRs) from the yeast genome. We analyzed genomic loci for which we had data for all 11 acetylated sites. In addition 
A

A

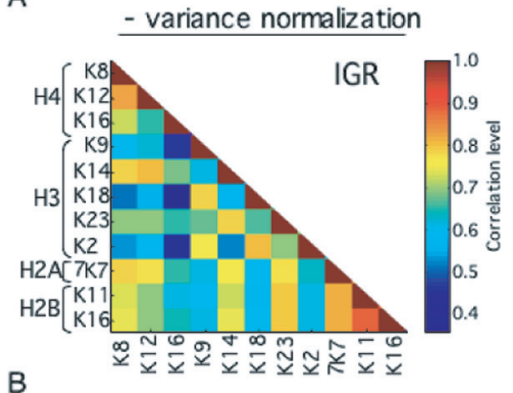

B

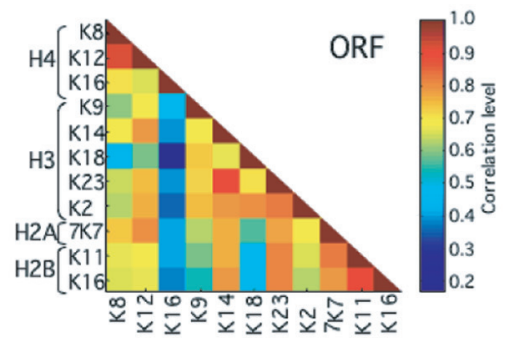

C

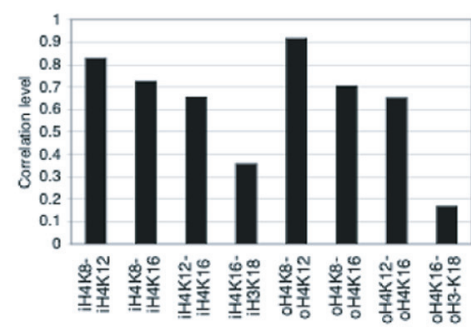

D

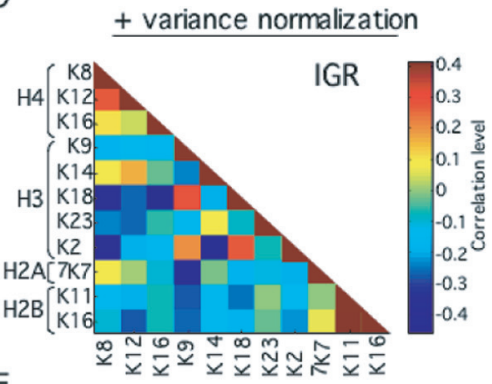

E

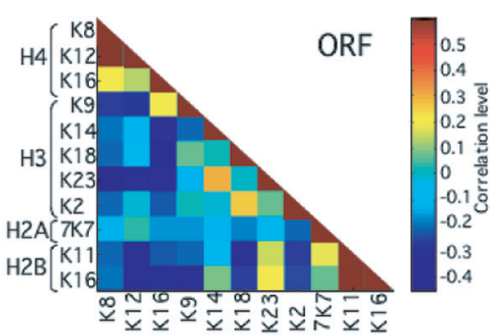

$\mathrm{F}$

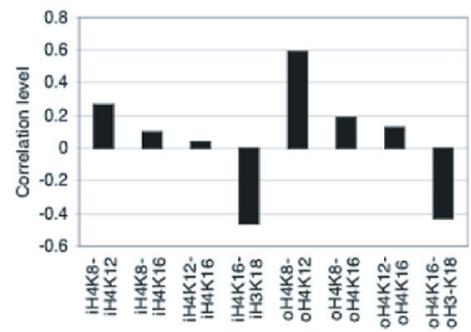

Figure 2. Correlation of acetylation levels at individual lysine residues with each other. The matrices $(A, B, D, E)$ show the degree of correlation (indicated by the color scale) between acetylation levels of different lysines. The diagonal is the correlation of each site to itself. $(A, B)$ Data from IGR and ORF arrays without variance normalization. (C) Correlation values for the acetylation levels of H4 lysine pairs on IGR ("i") and ORF ("o") arrays, depicted as bar graphs. The correlation of H4-K16 and H3-K18 is also shown. (D, $E)$ Correlation between acetylation levels of different sites after variance normalization of the acetylation profile across the 11 sites. These values reflect the relative acetylation level of each site compared to all other sites. $(F)$ Correlation values for the variance-normalized acetylation levels of $\mathrm{H} 4$ lysine pairs and $\mathrm{H} 4-\mathrm{K} 16 / \mathrm{H} 3-\mathrm{K} 18$, depicted as bar graphs. $(A, B, D, E$, Reprinted, with permission, from Kurdistani et al. 2004 [@Cell Press].)

to comparing the acetylation levels of individual lysines, we variance-normalized the data across the acetylation sites to compare relative acetylation levels of each lysine to all other lysines (for a more detailed description of methods and normalization procedures, see Kurdistani et al. 2004). Variance normalization emphasizes the local relationship among the various lysines rather than the more global (background) acetylation levels.

The correlation values obtained, before and after variance normalization, when the 11 acetylated sites were compared in a pair-wise manner are shown in Figure 2. Overall, acetylated lysines are positively correlated with each other. This means that, for a particular genomic region, if a single site is acetylated the probability of acetylation at other sites is high. In contrast to this general trend, the acetylation of H4-K16 has a distinctly lower correlation with other sites (Fig. 2A-C), and it is evident after variance normalization that H4-K16 acetylation is, in fact, negatively correlated with most other sites (Fig. 2D-F). In particular, H4-K16 is strongly anticorrelated with H3-K18 (Fig. 2F). H4-K16 acetylation correlates best with the other two $\mathrm{H} 4$ sites that were examined, $\mathrm{K} 8$ and K12 (Fig. 2C), although after variance normalization it is clear that $\mathrm{K} 16$ acetylation correlates poorly even with these two sites (Fig. 2F), mirroring the discrete functions of $\mathrm{K} 16$ and the other $\mathrm{H} 4$ acetylation sites. We believe that the high correlations we see before variance normalization are due to the pervasive global acetylation and deacetylation of lysines (Kuo et al. 2000; Vogelauer et al. 2000; Waterborg 2001), which would mask smaller local changes given the low resolution of the current generation of DNA microarrays.

\section{ACETYLATION AND TRANSCRIPTIONAL ACTIVITY}

Historically, hyperacetylation has been associated with transcription, while inactive regions have been characterized as hypoacetylated. This view is too simplistic, however, given the finding that the HDAC Hos2 acts prefer- 

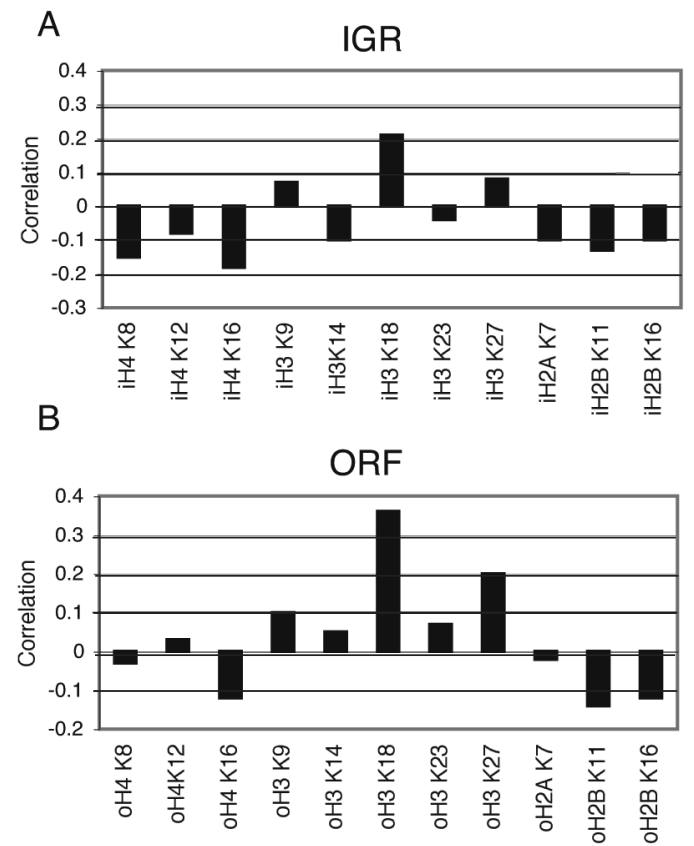

Figure 3. Correlation values between transcriptional activity and variance-normalized lysine acetylation levels on $(A)$ IGRs and $(B)$ ORFs.

entially on the coding regions of active genes genomewide, and is, in fact, required for gene activation (Wang et al. 2002). In order to assess how acetylation of particular lysines is correlated with gene expression, we compared our acetylation data with data on the transcriptional activity of genes throughout the genome under the experimental conditions we used (Causton et al. 2001). For both IGRs and ORFs, we conclude that the sites for which acetylation is best correlated with gene activity are on $\mathrm{H} 3$ (K18, followed by K9 and K27; Fig. 3A,B). Acetylation of lysines on H2A, H2B, and H4, on the other hand, show either a low or negative correlation with transcription. Notably, the acetylation of H2B sites K11 and K16, as well as H4-K16, shows a marked anticorrelation with active genes, lending further weight to the idea that deacetylation, as well as acetylation, can be important for normal gene activity.

\section{GENE CLUSTERS WITH SIGNATURE ACETYLATION PATTERNS}

The above correlations compare acetylation states for individual lysines with gene expression throughout the genome. The positive and negative correlations between acetylation sites and gene activity suggest that combinations of acetylated and deacetylated lysines may generate distinct modification patterns, each of which may be common to genes with related functions. To address this question systematically and without bias, we applied the $k$-means partitional clustering method (Hartigan 1975) to the variance-normalized acetylation data. Each IGR or ORF is included in only one cluster, based solely on the similarity of its acetylation patterns to other cluster mem- bers (Fig. 4A,C). In any one cluster, the correlation between patterns of acetylation for genes is 0.7 or higher. In this manner, we identified 53 and 68 groups of genes from IGRs and ORFs, respectively. Examination of the average acetylation patterns of the IGR clusters reveals a diverse array of acetylation patterns on the promoter regions. Remarkably, independent clustering of the ORF acetylation data also uncovered groups of genes with patterns as diverse as those of IGRs. We have shown by four different criteria that the genes in the acetylation clusters are biologically related to each other. Genes in the same cluster show significant coexpression, are significantly grouped within the same functional classes or physiological processes, have common upstream DNA sequence motifs, and show significant enrichment for binding of particular transcription factors (Kurdistani et al. 2004).

We find that the whole-genome relationships between individual lysines and their correlations to gene activity are further amplified in the acetylation clusters. This is perhaps due to the fact that these clusters are comprised of biologically cohesive groups of genes, and thus have a better signal to noise ratio. For instance, we find that H4$\mathrm{K} 16$ is hypoacetylated and $\mathrm{H} 3-\mathrm{K} 18$ is hyperacetylated in IGR cluster 1 and, conversely, in IGR cluster 30, H3-K18 is hypoacetylated and H4-K186 is hyperacetylated (Fig. 4B). Consistent with the genome-wide correlations, cluster 1 is highly expressed whereas cluster 30 is highly repressed under the conditions of growth used. The same is true for ORF clusters 1 and 6 (Fig. 4D). In fact, the inverse acetylation states of H4-K16 and H3-K18 are correlated strongly with expression state for a number of different clusters, indicating that, both globally and in the identified clusters, H3-K18 and H4-K16 are acetylated in an inverse manner with respect to each other. Therefore, for many clusters, the acetylation state of these sites can be a mark for transcriptional status. It will be interesting to determine whether such a mark exists in other eukaryotes as well.

\section{HOW IS K16 ACETYLATION REGULATED DURING GENE ACTIVITY?}

The acetylation and deacetylation of H4-K16 must be achieved by the enzymes that show specificity for this site within H4, making Esa1and Sas2 the candidate HATs and Rpd 3 and Hos2 the potential HDACs that target K16 in euchromatin (Allard et al. 1999; Kimura et al. 2002; Robyr et al. 2002; Suka et al. 2002). Rpd3 can be recruited to promoters by transcription factors such as Ume6, but also binds and deacetylates chromatin globally (Vogelauer et al. 2000; Kurdistani et al. 2002). Hos2, on the other hand, acts primarily on the coding region of active genes (Wang et al. 2002). While Rpd3 is strongly required for the deacetylation of $\mathrm{H} 4-\mathrm{K} 5, \mathrm{~K} 8$, and $\mathrm{K} 12$, Rpd3 deletion has the least effect on H4-K16 (Wu et al. 2001). Interestingly, the effect of Hos 2 deletion on K16 acetylation is greater than that of Rpd3 (Wang et al. 2002). Since Hos 2 acts on active coding regions, we must consider the possibility that this enzyme is responsible for the observed underacetylation of K16 on active genes 

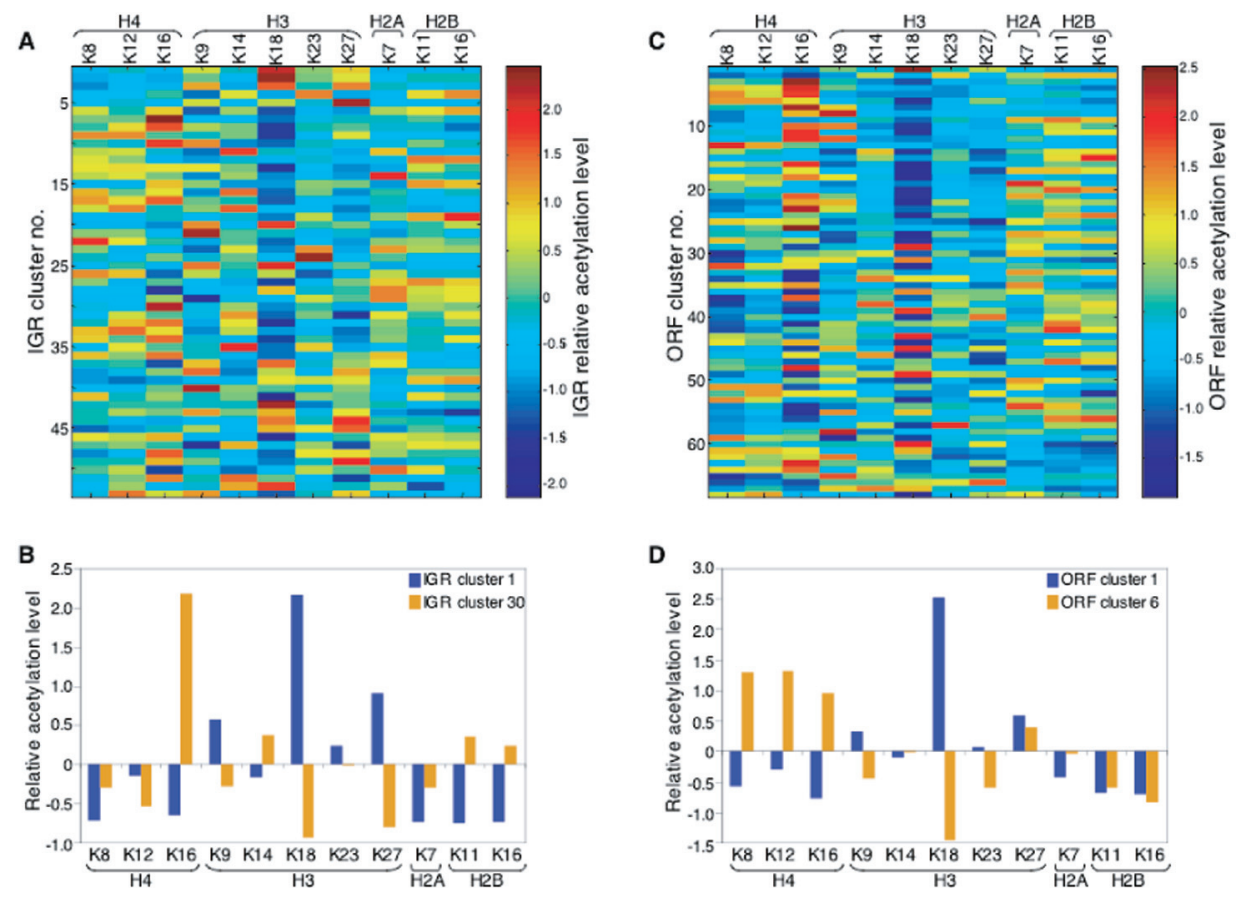

Figure 4. Groups of genes clustered based on their acetylation profile. (A) Average acetylation patterns of genes in the IGR clusters. Each row represents one cluster and, for each cluster, the average acetylation of each lysine is color-coded according to the scale shown. (B) Average acetylation patterns of genes in IGR clusters 1 and 30 as bar graphs. $(C)$ Average acetylation patterns of genes in the ORF clusters presented as in $A$. (D) Average acetylation patterns of genes in ORF clusters 1 and 6 as bar graphs. (Reprinted, with permission, from Kurdistani et al. 2004 [@Cell Press].)

genome-wide. Conversely, by acetylating K16, Esa1 and/or Sas2 may negatively regulate gene activity.

\section{ACETYLATION OF THE HISTONE H4 TAIL AND BDF1 BINDING}

The acetylation microarrays give us a picture of the genome-wide patterns of acetylation in logarithmically growing yeast cells. We wondered whether this information could be used to predict the binding preferences of chromatin proteins whose interaction with histones is affected by acetylation. Because the bromodomain has been identified as an acetyl-lysine binding domain, we looked at the genome-wide binding of Bdf1, a chromosomal protein that has two bromodomains and that binds preferentially to increasingly acetylated isoforms of H4 in vitro (Chua and Roeder 1995; Ladurner et al. 2003; Matangkasombut and Buratowski 2003).

Comparison of the binding profile of HA-tagged Bdf1 to the individual acetylation sites indicates that Bdf1 binding correlates positively with acetylation at all sites examined (Fig. 5A). The poorest correlation was with H4-K16 acetylation on ORFs. When the acetylation levels of the 11 lysines are normalized to each other (variance-normalized) and then compared to the genome-wide Bdf1 binding, the correlation with most sites remained positive, apart from H3-K9 and K27, H2B-K11, and H4K16. Relative acetylation of H4-K16 showed the strongest anticorrelation with Bdf1 binding (Fig. 5B).
Since Bdf1 is known to interact with the acetylated H4 tail, we looked in vivo at the consequences of point mutations in the $\mathrm{H} 4$ tail on Bdf1 binding (Fig. 5C,D). We found that mutation of $\mathrm{K} 12$ to arginine reduced binding at three out of the four promoters tested, arguing that, as predicted from the in vitro data, acetylation of H4-K12 is important for Bdf1 binding. In contrast, mutation of K16 to arginine had little effect on Bdf1 binding, suggesting that $\mathrm{K} 16$ acetylation is not required for Bdf1 binding at these promoters. In fact, substitution of K16 by glutamine (approximating the acetylated state) strongly reduced binding of Bdf1 to the four promoters in vivo. We conclude that optimal binding of Bdf1 to these promoters requires a positive charge at $\mathrm{H} 4$ site $\mathrm{K} 16$, indicating that the specific deacetylation of K16 may enhance Bdf1 binding to the otherwise acetylated $\mathrm{H} 4$ tail.

\section{H4-K16 ACETYLATION/DEACETYLATION IS A SWITCH FOR PROTEIN BINDING}

The amino terminus of $\mathrm{H} 4$ can be bound by a number of chromosomal proteins, and the acetylation status of $\mathrm{K} 16$ is a key regulator of these interactions. In most cases documented so far, deacetylation of K16 is a prerequisite for protein binding, but this deacetylation can affect binding to different subdomains in the H4 tail (Fig. 6). Sir3 binds the region carboxy-terminal to unacetylated K16 in the histone H4 tail (Fig. 6A). This region consists of a highly basic region (residues 16-19) and a relatively un- 
A

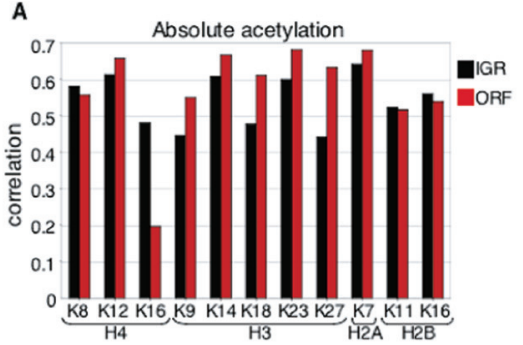

C

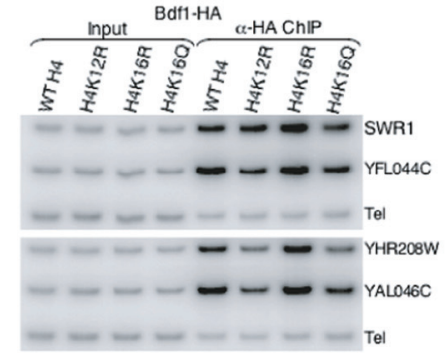

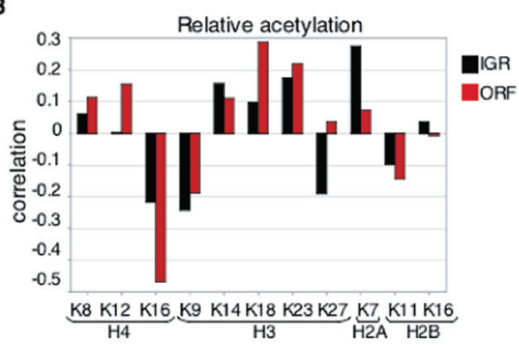

D

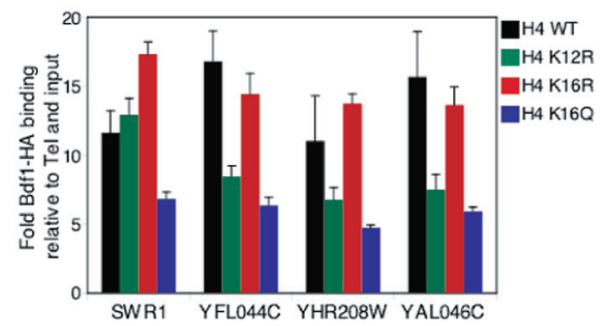

Figure 5. Genome-wide Bdf1 binding correlates with hypoacetylated H4-K16. (A) Bdf1 binding shows significant correlations with individual sites of acetylation on IGR (black bars) and ORF (red bars) regions, indicating that it binds to generally hyperacetylated regions of the genome. $(B)$ Correlation of Bdfl binding levels with variance-normalized acetylation levels. $(C, D)$ Effect of mutations in histone $\mathrm{H} 4$ on Bdf1 binding in vivo at the promoter regions of four randomly selected Bdf1-target genes. The intensity of Bdf1 enrichment is normalized to a region $500 \mathrm{bp}$ from the end of chromosome VI-R (Tel), which acts as an internal loading control. The fold binding is the ratio of Tel-normalized values of immunoprecipitated DNA to its input, averaged across three independent ChIP experiments. A representative gel is shown in $C$, and a bar graph plotting the enrichment values is shown in $D$. (K-R) Lysine to arginine substitution; (K-Q) lysine to glutamine mutation. (Reprinted, with permission, from Kurdistani et al. 2004 [@Cell Press].)

charged domain (residues 21-29), both of which are required for Sir3 binding in vivo and in vitro (Johnson et al. 1990, 1992; Hecht et al. 1995, 1996). The Drosophila remodeling factor ISWI has similar binding characteristics to Sir3. ISWI is also dependent on residues 16-19 of the H4 tail for its interaction with chromatin (Hamiche et al. 2001; Clapier et al. 2002) and acetylation of K16 reduces the ATPase activity of ISWI (Fig. 6B) (Clapier et al. 2002; Corona et al. 2002). The SLIDE (SANT-likeISWI-domain) is responsible for the interaction between ISWI and this region of H4 (Grune et al. 2003). Unexpectedly, K16 deacetylation is also favorable for the binding of the euchromatic bromodomain protein Bdf1. In this case, however, the acetylation of the other H4 amino-terminal lysines is also critical, indicating that K16 acetylation status may be important for the binding of Bdf1 to the region that is amino-terminal to K16 (Fig. $6 \mathrm{C})$. Because the deacetylation of K16 is important for Sir3, ISWI, and Bdf1 binding, it is possible that K16 deacetylation enhances the accessibility of the entire amino terminus of $\mathrm{H} 4$ in vivo, while acetylation of K16 restricts access. However, at least one protein is known to depend on K16 acetylation for its interaction with the H4 amino terminus. The crystal structure of the Gen5 bromodomain complexed with residues 15-29 of H4 shows that Gcn 5 makes contacts with the same region of the H4 tail involved in Sir3 binding (Owen et al. 2000), but K16 acetylation is favorable for binding in this case (Fig. 6D). It will be interesting to see if Gen5, or other factors, rely on K16 acetylation in order to interact with the H4 aminoterminal tail in vivo.
A

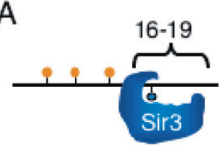

C
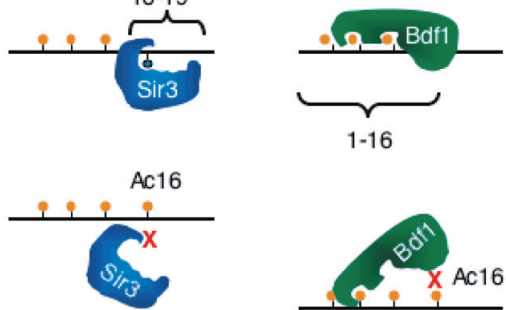

B

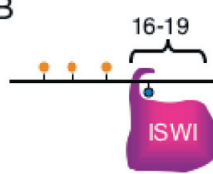

D
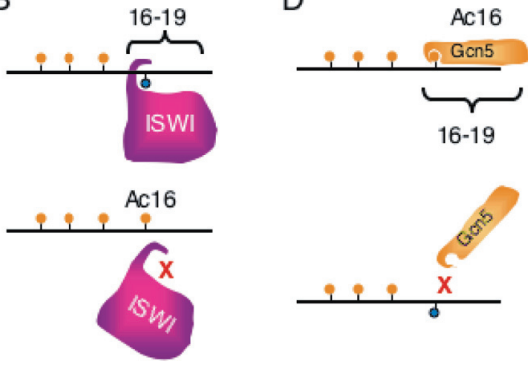

Figure 6. The acetylation state of H4-K16 controls the binding of multiple protein regulators. The $\mathrm{H} 4$ amino terminus is shown as a black line, with lysines 5,8,12, and 16 indicated as lollipops (blue for acetylated; orange for unacetylated). (A) Sir3 interacts with a region of $\mathrm{H} 4$ (residues 16-19) including K16. Acetylation of K16 decreases this interaction. (B) ISWI also interacts with residues $16-19$ of $\mathrm{H} 4$ when $\mathrm{K} 16$ is deacetylated. (C) Bdf1 binding is favored by K16 deacetylation, but acetylation of other sites is important, indicating that Bdf1 interacts with the region amino-terminal to K16. (D) Gen5 makes contacts with residues $16-19$ of $\mathrm{H} 4$ in vitro, but this interaction, unlike those in $A-C$, is favored by acetylation of K16. 
In conclusion, the acetylation state of H4-K16 may act a switch that determines the binding of numerous regulatory factors to different $\mathrm{H} 4$ regions, amino- or carboxyterminal to K16. The ability of K16 acetylation and deacetylation to affect multiple protein interactions with the $\mathrm{H} 4$ amino terminus may explain the unique status of K16 in the regulation of both heterochromatin and euchromatin.

\section{ACKNOWLEDGMENTS}

We thank Mona Shahbazian for helpful comments on the manuscript. C.B.M. is a recipient of a Wellcome Trust International Research Fellowship (ref: 069856). S.K.K. is a Howard Hughes Medical Institute Physician Postdoctoral Fellow. This work was supported by Public Service grants of NIH to M.G.

\section{REFERENCES}

Akhtar A. and Becker P.B. 2000. Activation of transcription through histone $\mathrm{H} 4$ acetylation by MOF, an acetyltransferase essential for dosage compensation in Drosophila. Mol. Cell 5: 367.

Allard S., Utley R.T., Savard J., Clarke A., Grant P., Brandl C.J., Pillus L., Workman J.L., and Cote J. 1999. NuA4, an essential transcription adaptor/histone $\mathrm{H} 4$ acetyltransferase complex containing Esalp and the ATM-related cofactor Tralp. EMBO J. 18: 5108.

Aparicio O.M., Billington B.L., and Gottschling D.E. 1991. Modifiers of position effect are shared between telomeric and silent mating-type loci in S. cerevisiae. Cell 66: 1279.

Bird A.W., Yu D.Y., Pray-Grant M.G., Qiu Q., Harmon K.E., Megee P.C., Grant P.A., Smith M.M., and Christman M. 2002. Acetylation of histone $\mathrm{H} 4$ by Esal is required for DNA double-strand break repair. Nature 419: 411.

Boyer L.A., Langer M.R., Crowley K.A., Tan S., Denu J.M., and Peterson C.L. 2002. Essential role for the SANT domain in the functioning of multiple chromatin remodeling enzymes. Mol. Cell 10: 935.

Carmen A.A., Milne L., and Grunstein M. 2002. Acetylation of the yeast histone $\mathrm{H} 4 \mathrm{~N}$ terminus regulates its binding to heterochromatin protein SIR3. J. Biol. Chem. 277: 4778.

Causton H.C., Ren B., Koh S.S., Harbison C.T., Kanin E., Jennings E.G., Lee T.I., True H.L., Lander E.S., and Young R.A. 2001. Remodeling of yeast genome expression in response to environmental changes. Mol. Biol. Cell 12: 323.

Chua P. and Roeder G.S. 1995. Bdf1, a yeast chromosomal protein required for sporulation. Mol. Cell. Biol. 15: 3685.

Clapier C.R., Nightingale K.P., and Becker P.B. 2002. A critical epitope for substrate recognition by the nucleosome remodeling ATPase ISWI. Nucleic Acids Res. 30: 649.

Clarke D.J., O'Neill L.P., and Turner B.M. 1993. Selective use of $\mathrm{H} 4$ acetylation sites in the yeast Saccharomyces cerevisiae. Biochem. J. 294: 557.

Corona D.F., Clapier C.R., Becker P.B., and Tamkun J.W. 2002. Modulation of ISWI function by site-specific histone acetylation. EMBO Rep. 3: 242.

Dhalluin C., Carlson J.E., Zeng L., He C., Aggarwal A.K., and Zhou M.M. 1999. Structure and ligand of a histone acetyltransferase bromodomain. Nature 399: 491.

Durrin L.K., Mann R.K., Kayne P.S., and Grunstein M. 1991. Yeast histone $\mathrm{H} 4 \mathrm{~N}$-terminal sequence is required for promoter activation in vivo. Cell 65: 1023.

Grune T., Brzeski J., Eberharter A., Clapier C.R., Corona D.F., Becker P.B., and Muller C.W. 2003. Crystal structure and functional analysis of a nucleosome recognition module of the remodeling factor ISWI. Mol. Cell 12: 449.

Hamiche A., Kang J.G., Dennis C., Xiao H., and Wu C. 2001. Histone tails modulate nucleosome mobility and regulate
ATP-dependent nucleosome sliding by NURF. Proc. Natl. Acad. Sci. 98: 14316.

Hartigan J. 1975. Clustering algorithms. Wiley, New York.

Hecht A., Strahl-Bolsinger S., and Grunstein M. 1996. Spreading of transcriptional repressor SIR3 from telomeric heterochromatin. Nature 383: 92.

Hecht A., Laroche T., Strahl-Bolsinger S., Gasser S.M., and Grunstein M. 1995. Histone H3 and H4 N-termini interact with SIR3 and SIR4 proteins: A molecular model for the formation of heterochromatin in yeast. Cell 80: 583 .

Hoppe G.J., Tanny J.C., Rudner A.D., Gerber S.A., Danaie S., Gygi S.P., and Moazed D. 2002. Steps in assembly of silent chromatin in yeast: Sir3-independent binding of a Sir2/Sir4 complex to silencers and role for Sir2-dependent deacetylation. Mol. Cell. Biol. 22: 4167.

Iyer V.R., Horak C.E., Scafe C.S., Botstein D., Snyder M., and Brown P.O. 2001. Genomic binding sites of the yeast cell-cycle transcription factors SBF and MBF. Nature 409: 533.

Jacobson R.H., Ladurner A.G., King D.S., and Tjian R. 2000. Structure and function of a human TAFII250 double bromodomain module. Science 288: 1422.

Johnson L.M., Kayne P.S., Kahn E.S., and Grunstein M. 1990. Genetic evidence for an interaction between SIR3 and histone $\mathrm{H} 4$ in the repression of the silent mating loci in Saccharomyces cerevisiae. Proc. Natl. Acad. Sci. 87: 6286.

Johnson L.M., Fisher-Adams G., and Grunstein M. 1992. Identification of a non-basic domain in the histone $\mathrm{H} 4 \mathrm{~N}$-terminus required for repression of the yeast silent mating loci. EMBO J. 11: 2201 .

Kasten M., Szerlong H., Erdjument-Bromage H., Tempst P., Werner M., and Cairns B.R. 2004. Tandem bromodomains in the chromatin remodeler RSC recognize acetylated histone H3 Lys14. EMBO J. 23: 1348.

Kayne P.S., Kim U.J., Han M., Mullen J.R., Yoshizaki F., and Grunstein M. 1988. Extremely conserved histone H4 N terminus is dispensable for growth but essential for repressing the silent mating loci in yeast. Cell 55: 27.

Kimura A., Umehara T., and Horikoshi M. 2002. Chromosomal gradient of histone acetylation established by Sas $2 p$ and Sir2p functions as a shield against gene silencing. Nat. Genet. 32: 370.

Kristjuhan A., Wittschieben B.O., Walker J., Roberts D., Cairns B.R., and Svejstrup J.Q. 2003. Spreading of Sir3 protein in cells with severe histone H3 hypoacetylation. Proc. Natl. Acad. Sci. 100: 7551.

Kuo M.H., vom Baur E., Struhl K., and Allis C.D. 2000. Gen4 activator targets Gen5 histone acetyltransferase to specific promoters independently of transcription. Mol. Cell 6: 1309.

Kurdistani S.K., Tavazoie S., and Grunstein M. 2004. Mapping global histone acetylation patterns to gene expression. Cell 117: 721.

Kurdistani S.K., Robyr D., Tavazoie S., and Grunstein M. 2002. Genome-wide binding map of the histone deacetylase Rpd3 in yeast. Nat. Genet. 31: 248.

Ladurner A.G., Inouye C., Jain R., and Tjian R. 2003. Bromodomains mediate an acetyl-histone encoded antisilencing function at heterochromatin boundaries. Mol. Cell 11: 365 .

Lenfant F., Mann R.K., Thomsen B., Ling X., and Grunstein M. 1996. All four core histone N-termini contain sequences required for the repression of basal transcription in yeast. EMBO J. 15: 3974.

Luger K., Mader A.W., Richmond R.K., Sargent D.F., and Richmond T.J. 1997. Crystal structure of the nucleosome core particle at $2.8 \AA$ Aresolution. Nature 389: 251.

Luo K., Vega-Palas M.A., and Grunstein M. 2002. Rap1-Sir4 binding independent of other $\mathrm{Sir}, \mathrm{yKu}$, or histone interactions initiates the assembly of telomeric heterochromatin in yeast. Genes Dev. 16: 1528.

Ma X.J., Wu J., Altheim B.A., Schultz M.C., and Grunstein M. 1998. Deposition-related sites K5/K12 in histone H4 are not required for nucleosome deposition in yeast. Proc. Natl. Acad. Sci. 95: 6693.

Matangkasombut O. and Buratowski S. 2003. Different sensitivities of bromodomain factors 1 and 2 to histone $\mathrm{H} 4$ acetyla- 
tion. Mol. Cell 11: 353.

Megee P.C., Morgan B.A., Mittman B.A., and Smith M.M. 1990. Genetic analysis of histone H4: Essential role of lysines subject to reversible acetylation. Science 247: 841.

Meneghini M.D., Wu M., and Madhani H.D. 2003. Conserved histone variant $\mathrm{H} 2 \mathrm{~A} . \mathrm{Z}$ protects euchromatin from the ectopic spread of silent heterochromatin. Cell 112: 725.

$\mathrm{Ng} \mathrm{H.H.,} \mathrm{Dole} \mathrm{S.,} \mathrm{and} \mathrm{Struhl} \mathrm{K.} \mathrm{2003.} \mathrm{The} \mathrm{Rtf1} \mathrm{component} \mathrm{of}$ the Pafl transcriptional elongation complex is required for ubiquitination of histone H2B. J. Biol. Chem. 278: 33625.

Owen D.J., Ornaghi P., Yang J.C., Lowe N., Evans P.R., Ballario P., Neuhaus D., Filetici P., and Travers A.A. 2000. The structural basis for the recognition of acetylated histone $\mathrm{H} 4$ by the bromodomain of histone acetyltransferase Gen5p. EMBO J. 19: 6141.

Park E.C. and Szostak J.W. 1990. Point mutations in the yeast histone $\mathrm{H} 4$ gene prevent silencing of the silent mating type locus HML. Mol. Cell. Biol. 10: 4932.

Ren B., Robert F., Wyrick J.J., Aparicio O., Jennings E.G., Simon I., Zeitlinger J., Schreiber J., Hannett N., Kanin E., Volkert T.L., Wilson C.J., Bell S.P., and Young R.A. 2000. Genome-wide location and function of DNA binding proteins. Science 290: 2306.

Robyr D., Suka Y., Xenarios I., Kurdistani S.K., Wang A., Suka N., and Grunstein M. 2002. Microarray deacetylation maps determine genome-wide functions for yeast histone deacetylases. Cell 109: 437.

Sabet N., Tong F., Madigan J.P., Volo S., Smith M.M., and Morse R.H. 2003. Global and specific transcriptional repression by the histone $\mathrm{H} 3$ amino terminus in yeast. Proc. Natl. Acad. Sci. 100: 4084.

Smith C.M., Gafken P.R., Zhang Z., Gottschling D.E., Smith J.B., and Smith D.L. 2003. Mass spectrometric quantification of acetylation at specific lysines within the amino-terminal tail of histone H4. Anal. Biochem. 316: 23.
Suka N., Luo K., and Grunstein M. 2002. Sir2p and Sas2p opposingly regulate acetylation of yeast histone H4 lysine16 and spreading of heterochromatin. Nat. Genet. 32: 378 .

Suka N., Suka Y., Carmen A.A., Wu J., and Grunstein M. 2001. Highly specific antibodies determine histone acetylation site usage in yeast heterochromatin and euchromatin. Mol. Cell 8: 473.

Tse C., Sera T., Wolffe A.P., and Hansen J.C. 1998. Disruption of higher-order folding by core histone acetylation dramatically enhances transcription of nucleosomal arrays by RNA polymerase III. Mol. Cell. Biol. 18: 4629.

van Leeuwen F., Gafken P.R., and Gottschling D.E. 2002. Dot $1 \mathrm{p}$ modulates silencing in yeast by methylation of the nucleosome core. Cell 109: 745.

Vogelauer M., Wu J., Suka N., and Grunstein M. 2000. Global histone acetylation and deacetylation in yeast. Nature 408: 495.

Wang A., Kurdistani S.K., and Grunstein M. 2002. Requirement of Hos2 histone deacetylase for gene activity in yeast. Science 298: 1412.

Waterborg J.H. 2001. Dynamics of histone acetylation in Saccharomyces cerevisiae. Biochemistry 40: 2599.

Wu J., Suka N., Carlson M., and Grunstein M. 2001. TUP1 utilizes histone $\mathrm{H} 3 / \mathrm{H} 2 \mathrm{~B}$-specific HDA1 deacetylase to repress gene activity in yeast. Mol. Cell 7: 117.

Yu J., Li Y., Ishizuka T., Guenther M.G., and Lazar M.A. 2003. A SANT motif in the SMRT corepressor interprets the histone code and promotes histone deacetylation. $E M B O J .22$ : 3403.

Zhang L., Eugeni E.E., Parthun M.R., and Freitas M.A. 2003. Identification of novel histone post-translational modifications by peptide mass fingerprinting. Chromosoma 112: 77.

Zheng C. and Hayes J.J. 2003. Intra- and inter-nucleosomal protein-DNA interactions of the core histone tail domains in a model system. J. Biol. Chem. 278: 24217. 


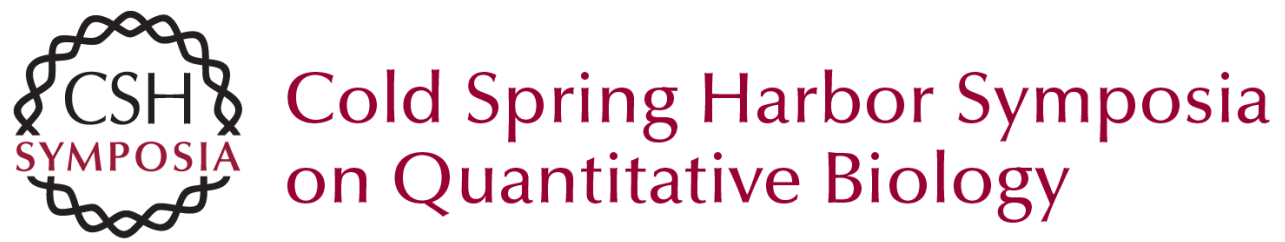

\section{Acetylation of Yeast Histone H4 Lysine 16: A Switch for Protein Interactions in Heterochromatin and Euchromatin}

C.B. MILLAR, S.K. KURDISTANI and M. GRUNSTEIN

Cold Spring Harb Symp Quant Biol 2004 69: 193-200

Access the most recent version at doi:10.1101/sqb.2004.69.193

References This article cites 55 articles, 23 of which can be accessed free at: http://symposium.cshlp.org/content/69/193.full.html\#ref-list-1

\section{License}

Email Alerting

Receive free email alerts when new articles cite this article - sign up in Service the box at the top right corner of the article or click here. 